etc., these being of weight 4 and 6 respectively.

If the system of equations (2) is equivalent to a single linear differential equation of the second order, all of the invariants vanish, and conversely.

Any system of form (2) can be transformed into another for which $p_{i k}=0$. 'This is called the semicanonical form of the system. The subgroup $G^{\prime}$ of $G$ which leaves this form unchanged is examined. But we can fulfill the further condition $q_{11}+q_{22}=0$, and a system for which both $p_{i k}=0$ and $q_{11}+q_{22}=0$, is said to be in the canonical form. The subgroup $G^{\prime \prime}$ of $G^{\prime}$ which leaves this unchanged is a finite group of very simple form, and has some additional invariants of the form called quadri-derivatives by Forsyth.

Only a few simple results about covariants are mentioned. This and further generalizations are left for a future paper.

Columbia University.

F. N. Cole.

\title{
ON LINEAR DEPENDENCE OF FUNCTIONS OF
} ONE VARIABLE.

BY PROFESSOR MAXIME BôCHER.

It is ordinarily stated that the identical vanishing of the determinant

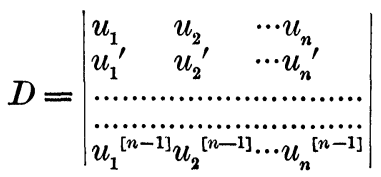

is a sufficient $*$ condition for the linear dependence of the functions $u_{1}(x), u_{2}(x), \cdots, u_{n}(x)$. This is perfectly true if the $u$ 's are analytic functions of the complex variable $x$. This condition is however no longer sufficient if we are dealing with functions of a real variable, even though these functions possess derivatives of all orders for every real value of $x$. The truth of this statement will be seen from the example of two functions $u_{1}$ and $u_{2}$ defined as follows:

$$
u_{1}=\left\{\begin{array}{ll}
e^{-1 / x^{2}} & (x \neq 0) \\
0 & (x=0)
\end{array} \quad u_{2}= \begin{cases}e^{-1 / x^{2}} & (x>0) \\
0 & (x=0) \\
2 e^{-1 / x^{2}} & (x<0)\end{cases}\right.
$$

* It is of course a necessary condition provided the $u$ 's have derivatives of the first $n-1$ orders. 
Similar examples for a larger number of functions can readily be built.

The following theorem however is true :

If $u_{1}, u_{2}, \cdots, u_{n}$ are single valued functions of the real variable $x$ defined at every point of a certain interval and having at every point of this interval derivatives of the first $n-1$ orders, and if it is possible to strike out the last row and one of the columns of the determinant $D$ in such a way that there is no point of the interval in question at which the remaining determinant and its derivative both vanish, then if $D$ vanishes at every point of the interval, the functions $u_{1}, u_{2}, \cdots, u_{n}$ will be linearly dependent throughout this interval.

This theorem can be readily proved by a slight extension of the method given for instance by Heffter in his book on linear differential equations p. 233.

Ems, Germany,

September 15, 1900.

[Note added November 2, 1900: I have just found in Pascal's book on determinants a reference to three papers by Peano (Mathesis, vol. 9 (1889), p. 75 and p. 110 ; Rend. d. Accad. d. Lincei, ser. 5, vol. 6 (1897), $1^{\circ}$ sem., p. 413), in which the question which I have here considered is taken up. My result is however different from Peano's, which states that the identical vanishing of $D$ is a sufficient condition for linear dependence, provided there is no point at which the first minors corresponding to the elements of the last column all vanish.]

\section{REPORT ON THE GROUPS OF AN INFINITE ORDER.}

BY DR. G. A. MILLER.

(Read before Section A of the American Association for the Advancement of Science, New York, June 28, 1900.)

VARIous terms have been employed to designate the smallest elements of which any abstract group is composed. Cayley has called them symbols, * or symbols of operation. Dyck and many others have called them operations $\dagger$ or operators. Frobenius and others have called them elements. I In what follows we shall employ the last one of

\footnotetext{
* Cayley, Phil. Magazine, vol. 7 (1854), p. 41

† Dyck, Math. Annalen, vol. 20 (1882), p. 1.

$\ddagger$ Frobenius, Crelle, vol. 86 (1879), p. 218.
} 